\title{
Simultaneous HPLC determination of flavonoids and phenolic acids profile in Pêra-Rio orange juice
}

\author{
Mesquita E., Monteiro M.* \\ Department of Food and Nutrition, School of Pharmaceutical Science, São Paulo State University, UNESP, 14800-903 Araraquara, SP, Brazil
}

\section{A R T I C L E I N F O}

\section{Keywords:}

Phenolic compounds

HPLC-DAD

Validation

Organic orange juice

Flavonoids

Phenolic acids

\begin{abstract}
A B S T R A C T
The aim of this study was to develop and validate an HPLC-DAD method to evaluate the phenolic compounds profile of organic and conventional Pêra-Rio orange juice. The proposed method was validated for 10 flavonoids and 6 phenolic acids. A wide linear range (0.01-223.4 $\left.\mu \mathrm{g} \cdot \mathrm{g}^{-1}\right)$, good accuracy (79.5-129.2\%) and precision $(\mathrm{CV} \leq 3.8 \%)$, low limits of detection $\left(1-22 \mathrm{ng} \cdot \mathrm{g}^{-1}\right)$ and quantification $(0.7-7.4 \mu \mathrm{g})$, and overall ruggedness were attained. Good recovery was achieved for all phenolic compounds after extraction and cleanup. The method was applied to organic and conventional Pêra-Rio orange juices from beginning, middle and end of the 2016 harvest. Flavones rutin, nobiletin and tangeretin, and flavanones hesperidin, narirutin and eriocitrin were identified and quantified in all organic and conventional juices. Identity was confirmed by mass spectrometry. Nineteen non-identified phenolic compounds were quantified based on DAD spectra characteristic of the chemical class: 7 cinnamic acid derivatives, 6 flavanones and 6 flavones. The phenolic compounds profile of PêraRio orange juices changed during the harvest; levels increased in organic orange juices, and decreased or were about the same in conventional orange juices. Phenolic compounds levels were higher in organic $\left(0.5-1143.7 \mathrm{mg} \cdot 100 \mathrm{~g}^{-1}\right)$ than in conventional orange juices $\left(0.5-689.7 \mathrm{mg} \cdot 100 \mathrm{~g}^{-1}\right)$. PCA differentiated organic from conventional FS and NFC juices, and conventional FCOJ from conventional FS and NFC juices, thus differentiating cultivation and processing.
\end{abstract}

\section{Introduction}

Orange is the main fruit produced in Brazil, with ca 450 million boxes in 2017. The majority of oranges are delivered for NFC (Not From Concentrate) and FCOJ (Frozen Concentrated Orange Juice) production and exportation. Of the total 1.01 million ton juice produced in 2016, 921,000 ton were from São Paulo, of which 699,000 ton FCOJ and 222,000 ton NFC (FCOJ equivalent). The prediction is 848,000 ton in 2017, 771,000 from São Paulo; 531,000 ton FCOJ and 240,000 ton NFC (FCOJ equivalent). More than $80 \%$ of the juice is exported to Europe and the USA (Fundecitrus, 2017; Neves et al., 2010; USDA, 2016).

Organic citrus fruit represents $0.9 \%$ of the total worldly production; amongst it, oranges are the most cultivated fruit and orange juice is the main organic product. Latin America holds the biggest area of organic cultivation in the world (ca 15,000 ha), where Brazil has a strong position as a productor and a potential consumption market (FAO, 2003; IFOAM, 2015).

There is still very little knowledge about the influence of the cultivation system on food quality, despite the increasing consumption of organic foods especially by environment-friendly and health-conscious consumers (Janzantti, Santos, \& Monteiro, 2012; Macoris, De Marchi, Janzantti, \& Monteiro, 2011; Macoris, Janzantti, Garruti, \& Monteiro, 2011; Santos \& Monteiro, 2004). It is necessary to assess organic foods from the chemical, nutritional and sensory points of view, to evaluate the characteristics and verify the potential health benefits associated with consumption.

Orange juice consumption has been associated with health benefits, mainly related to modulation of the human metabolism, and antioxidant and anti-inflammatory activities, which prevent chronic-degenerative diseases such as cardiovascular diseases, diabetes and cancer. Orange juice is a dietary source of ascorbic acid, and also contains flavonoids and phenolic acids, which highly prevent oxidative stress (Barreca et al., 2017; Khan, Huma, \& Dangles, 2014; MedinaRemon, Estruch, Tresserra-Rimbau, Vallverdú-Queralt, \& LamuelaRaventos, 2013; Peterson et al., 2006; Tripoli, La Guardia, Giammanco, Di Majo, \& Giammanco, 2007).

The phenolic composition of orange juice is influenced by the variety and maturity of the fruit, edaphoclimatic conditions, cultivation system, post-harvest and processing conditions (Baldwin, Scott, Shewmaker, \& Schuch, 2000; Haard, 1984; Macoris, De Marchi, et al.,

\footnotetext{
* Corresponding author.

E-mail address: monteiro@fcfar.unesp.br (M. Monteiro).
} 
2011; Zou, Xi, Hu, Nie, \& Zhou, 2016). The main Citrus phenolic compounds are flavonoids and cinnamic acid derivatives (Gattuso, Barreca, Gargiulli, Leuzzi, \& Caristi, 2007; Khan et al., 2014; Peterson et al., 2006). Most of the methods for phenolic compounds analysis are based on HPLC technique in reversed phase and gradient mode, with DAD and/or MS detection. Several methods have been applied to honey (Escriche, Kadar, Juan-Borrás, \& Domenech, 2011), grapes (Burin, Ferreira-Lima, Panceri, \& Bordignon-Luiz, 2014), bayberry (Fang, Wang, Hao, \& Guo, 2009), dates (Abu-Reidah, Ali-Shtayeh, Jamous, Arráez-Román, \& Segura-Carretero, 2015), guava (Rojas-Garbanzo, Zimmermann, Schulze-Kaysers, \& Schieber, 2016), mango (López-Cobo, Gómez-Caravaca, Svarc-Gajíc, Segura-Carretero, \& FernándezGutiérrez, 2015), cherry (Martini, Conte, \& Tagliazucchi, 2017), strawberries (Pinto, Lajolo, \& Genovese, 2008) and tomato (VallverdúQueralt, Arranz, Medina-Remón, Casals-Ribes, \& Lamuela-Raventos, 2011; Vallverdú-Queralt, Jáuregui, Medina-Remon, \& LamuelaRaventos, 2012). Furthermore, flavonoids and phenolic acids have been reported in Kozan, Navel, Moro, Tarocco, Sanguinello, Salustiana, CaraCara, Baia, Natal and Lima orange juices. Hesperidin is by far the major flavonoid, followed by narirutin. Amongst the acids, ferulic, caffeic and coumaric acids have been reported in Kozan and Navel orange juices (Gattuso et al., 2007; Gil-Izquierdo, Gil, Ferreres, \& Tomás-Barberán, 2001; Kelebek, Selli, Canbas, \& Cabaroglu, 2009; Leuzzi, Caristi, Panzera, \& Licandro, 2000; Rapisarda et al., 1999).

Despite many studies on flavonoid composition in orange juice from several varieties, the Pêra-Rio variety was not included and there is still little knowledge about the compounds other than hesperidin and narirutin. Pêra-Rio is the most important variety cultivated in Brazil and is responsible for the uniqueness of the Brazilian juice. To the best of our knowledge, there is a lack of studies regarding the phenolic profile of orange juice, as well as the Pêra-Rio variety.

The aim of this work was to develop and validate an HPLC-DAD method for simultaneous flavonoid and phenolic acids profile analysis in Pêra-Rio orange juice from organic and conventional cultivation systems during the 2016 harvest.

\section{Material and methods}

\subsection{Chemicals}

Pure standards (95-99\%, Sigma Aldrich) of six phenolic acids (gallic, protocatechuic, caffeic, syringic, coumaric and ferulic acids) and ten flavonoids (rutin, eriocitrin, quercitrin, narirutin, naringin, hesperidin, naringenin, hesperitin, nobiletin, and tangeretin) were used, and benzoic acid was used as the internal standard.

Acetonitrile, methanol and ethyl acetate were of HPLC grade, formic acid was of analytical grade, and ultrapure water was obtained from a Direct Q-3 UV system (Millipore, USA).

\subsection{Orange juice samples}

Organic freshly-squeezed (FS) and NFC Pêra-Rio orange juices from the 2016 harvest were provided by a certified producer (no CA5897/15, IBD Certification) from Itirapina, SP, Brazil $\left(22^{\circ} 15^{\prime} 10^{\prime \prime} \mathrm{S}, 47^{\circ} 49^{\prime} 22^{\prime \prime}\right.$ W). Conventional FS, NFC and FCOJ Pêra-Rio orange juices were provided by a citrus industry from Araraquara region, SP, Brazil $\left(21^{\circ} 47^{\prime}\right.$ $\left.40^{\prime \prime} \mathrm{S}, 48^{\circ} 10^{\prime} 32^{\prime \prime} \mathrm{W}\right)$.

FS juices $(2 \mathrm{~L})$ were collected from the finishing step, NFC juices $(2 \mathrm{~L})$ from the pasteurization step and FCOJ $(500 \mathrm{~mL})$ from the concentration step. The collection of the juices from each step was performed from the same load of oranges, so that all the juice collected was from the same processing batch. All juices were sampled from July to October 2016 at the beginning, middle and end of harvest. Juices were frozen and lyophilized. Phenolic compounds were extracted immediately after lyophilization.

\subsection{Phenolic compounds extraction}

Lyophilized orange juice was weighted (3-4.0000 g) and added of a methanol aqueous solution $(90 \%, \mathrm{v} / \mathrm{v})$, homogenized (1 min) and extracted in ultrasonic bath $(20 \mathrm{~min})$, then centrifuged at $9000 \mathrm{rpm}$ at $20{ }^{\circ} \mathrm{C}(20 \mathrm{~min})$. Supernatant was collected and the extraction was repeated. Supernatants were combined and submitted to a cleanup step using solid phase extraction (SPE).

SPE conditions were evaluated employing conventional NFC juice. A standard solution of the phenolic compounds (3.106-23.368 $\mu \mathrm{g} \cdot \mathrm{g}^{-1}$ ) was added to the juice prior to the extraction for recovery evaluation. C18 (Bond Elut, Agilent Technologies, USA) and polymeric (Strata X, Phenomenex, USA) cartridges, both of $500 \mathrm{mg}$ phase and $6 \mathrm{~mL}$ volume, were conditioned with acetonitrile $(18 \mathrm{~mL})$ and aqueous formic acid $(0.1 \% \mathrm{v} / \mathrm{v})(18 \mathrm{~mL})$ or ethyl acetate $(18 \mathrm{~mL})$ and aqueous formic acid $(0.1 \%, \mathrm{v} / \mathrm{v})(18 \mathrm{~mL})$. Extracts were introduced in the cartridges and collected, and then cartridges were washed with acetonitrile $(30 \mathrm{~mL})$ or ethyl acetate $(30 \mathrm{~mL})$ for the elution of the retained compounds. Eluted extracts and solvents were combined, dried under nitrogen flow and reconstituted with aqueous formic acid $(0.1 \%, \mathrm{v} / \mathrm{v})$.

SPE conditions were selected based on peak area repeatability and recovery of phenolic compounds. C18 cartridges conditioned with ethyl acetate $(18 \mathrm{~mL})$ and aqueous formic acid $(18 \mathrm{~mL})$, and washed with ethyl acetate $(30 \mathrm{~mL})$ were selected. After drying and reconstitution as previously described, aqueous extracts were weighted, filtered through $0.22 \mu \mathrm{m}$ regenerated cellulose disk filters and stored at $-20{ }^{\circ} \mathrm{C}$ until analysis. Extracts were obtained in duplicate for each juice.

\subsection{HPLC-DAD and MS conditions}

Liquid chromatography was carried out in an Acquity ARC system (Waters, USA) with a diode array detector, using a BEH X-Bridge C18 column $(250 \times 4.6 \mathrm{~mm}, 5 \mu \mathrm{m})$ and guard column $(20 \times 4.6 \mathrm{~mm}$, $5 \mu \mathrm{m})$. For development purposes, mobile phase was water acidified with formic acid $(0-5 \%, \mathrm{v} / \mathrm{v})$ and acetonitrile or methanol. Column temperature $\left(25-50{ }^{\circ} \mathrm{C}\right)$, volume of injection $(5-20 \mu \mathrm{L})$ and flow rate $\left(0.7-1.0 \mathrm{~mL} \cdot \mathrm{min}^{-1}\right)$ conditions were tested and selected based on peak number, symmetry and resolution. Wavelengths were monitored from 210 to $400 \mathrm{~nm}$ and chromatograms were acquired at 255, 270 and $280 \mathrm{~nm}$. Final working conditions were: mobile phase of aqueous formic acid solution $(0.1 \%, \mathrm{v} / \mathrm{v})$ and acetonitrile, column temperature of $50{ }^{\circ} \mathrm{C}, 20 \mu \mathrm{L}$ of injection volume, flow rate of $1.0 \mathrm{~mL} \cdot \mathrm{min}^{-1}$ and gradient of $6-10 \%$ acetonitrile (0-16 min), $10-22 \%$ (16-36 min), $22-100 \%$ (36-38 $\mathrm{min}$ ) and held for $5 \mathrm{~min}$. Column was equilibrated for 10 min between injections.

An Acquity HPLC system (Waters, USA) with a single-quadrupole QDa mass detector using the same column and separation conditions as in HPLC-DAD was used. Electrospray ionization and MS analysis conditions were as follows: capillary voltage $1.5 \mathrm{kV}$ (positive mode) and $0.8 \mathrm{kV}$ (negative mode), probe temperature of $500{ }^{\circ} \mathrm{C}, \mathrm{N}_{2}$ as drying gas and MS Scan from 100 to $900 \mathrm{~m} / \mathrm{z}$. Mass spectra were obtained in both positive and negative ionization modes.

\subsection{Validation}

The method was validated using analytical figures of merit, based on international validation protocols (ICH, 2005; Magnusson \& Örnemark, 2014; Thompson, Ellison, \& Wood, 2002). Calibration, linearity, limit of detection, precision, accuracy, limit of quantification and ruggedness were evaluated.

\subsection{Phenolic compounds profile of Pêra-Rio orange juice}

Organic and conventional orange juice extracts were injected in HPLC-DAD system in triplicate. Phenolic compounds were identified based on retention time and DAD spectra. In order to confirm peak 
identities, orange juice extracts were injected in the HPLC-MS system. Pure standards of 10 flavonoids and 6 phenolic acids were used for comparison. For quantification purposes, ferulic acid, rutin, eriocitrin, narirutin, hesperidin, nobiletin and tangeretin calibration curves with 4 to 5 points $\left(0.041-73.787 \mu \mathrm{g} \cdot \mathrm{g}^{-1}\right)$ were prepared and injected in triplicate in HPLC-DAD system, using benzoic acid as the internal standard $\left(55 \mu g \cdot g^{-1}\right)$.

\subsection{Data analysis}

Principal component analysis (PCA) was carried out based on the correlation matrix of the phenolic compounds area values in each juice extract from organic and conventional cultivation systems during the harvest. Phenolic compounds were represented by the name, peak number and/or chemical class. Only variables with correlation (rotate component matrix) $\geq 0.60$ were considered. Groups were highlighted according to behavior in the cultivation systems and/or juices. PCA was performed using STATISTICA 10.0.

\section{Results and discussion}

\subsection{HPLC-DAD method development}

The method was developed based on methods previously described for phenolic analysis in orange and orange juice (Barreca, Bellocco, Caristi, Leuzzi, \& Gattuso, 2011; Bilbao, Andrés-Lacueva, Jáuregui, \& Lamuela-Raventos, 2007; Gil-Izquierdo et al., 2001; Kelebek et al., 2009; Leuzzi et al., 2000). Initially, an exploratory gradient from 5 to 95\% methanol was employed and injection volume, flow rate and temperature conditions were independently tested. Based on overall resolution, peak number and symmetry, an injection volume of $20 \mu \mathrm{L}$, flow rate of $1 \mathrm{~mL} \cdot \mathrm{min}^{-1}$ and temperature of $50{ }^{\circ} \mathrm{C}$ were selected. Then, mobile phase composition was varied and methanol was replaced by acetonitrile resulting in better peak symmetry and resolution. Simultaneously, water acidified with formic acid in different proportions was tested. No acid resulted in overall bad resolution, as well as too much acid, so $0.1 \%(\mathrm{v} / \mathrm{v})$ was chosen. Lastly, in order to attain better separation in specific portions of the chromatogram, adjustments were made in the gradient. For such, a low rate of organic solvent increase was employed in the beginning $\left(0.25 \% \cdot \mathrm{min}^{-1}\right)$ and middle $\left(0.60 \% \cdot \mathrm{min}^{-1}\right)$, and a faster rate at the end $\left(39 \% \cdot \mathrm{min}^{-1}\right)$, in a total run time of $43 \mathrm{~min}$. It was also necessary to adjust the initial and final proportions of acetonitrile resulting in a gradient of $6-10 \%$ acetonitrile from 0 to $16 \mathrm{~min}, 10-22 \%$ from 16 to $36 \mathrm{~min}, 22-100 \%$ from 36 to $38 \mathrm{~min}$, and held until $43 \mathrm{~min}$. Column was equilibrated for $10 \mathrm{~min}$ between injections. Based on compound structures and classes, wavelengths were monitored from 210 to $400 \mathrm{~nm}$ and chromatograms were acquired at 255, 270 and $280 \mathrm{~nm}$. Good resolution was attained, with a mean of 50 peaks with total area of approximately 15 million area units in the chromatogram.

\subsection{Phenolic compounds extraction from orange juice}

At the beginning of the experiment, the chromatogram of some orange juice extracts showed certain regions with a lack of sufficient resolution, which also affected the repeatability of some peak area values. These suggested that a more thorough extraction procedure should be performed, for which SPE was considered.

Polymeric (Strata X, Phenomenex) and C18 (Bond Elut, Agilent Technologies) cartridges conditioned and washed with different solvent combinations were tested. Repeatability of area values of the peaks was improved by SPE, as well as overall resolution in the chromatogram. Recovery of phenolic acids and flavonoids ranged from 9.4-104.0\% with acetonitrile and $80.1-101.9 \%$ with ethyl acetate for C18 cartridges, $33.7-81.7 \%$ with acetonitrile and $43.3-71.1 \%$ with ethyl acetate for polymeric cartridges, and $43.7-81.4 \%$ without SPE. An exception of $0 \%$ recovery for protocatechuic acid was obtained with C18 cartridges when acetonitrile was used. C18 cartridges showed overall better recovery and were therefore more suitable. Regarding solvents, ethyl acetate showed high recovery levels for all phenolic acids and flavonoids, whereas acetonitrile showed low recovery levels for gallic and protocatechuic acids, rutin, eriocitrin, quercitrin and narirutin. Ethyl acetate was then selected as the solvent for the conditioning of the cartridges and elution of phenolic compounds.

\subsection{HPLC-DAD method validation}

After method development, it was necessary to validate it. Validation was performed according to international guidelines (ICH, 2005; Magnusson \& Örnemark, 2014; Thompson et al., 2002). Calibration curves with four to five points were plotted for each compound, as well as area/concentration ratio versus concentration log for linearity evaluation. Limit of detection was calculated, intermediate precision and repeatability were evaluated in three levels of concentration for each compound, and accuracy was assessed using recovery in three levels of concentration for each compound. Limit of quantification was based on the recovery assay. Ruggedness was evaluated with variations on mobile phase, organic solvent proportion in the gradient and column temperature. Analytical figures of merit are shown in Table 1.

The developed method showed a wide linear range for the proposed application, good repeatability with RSD $\leq 3.6 \%$ for the three levels, and intermediate precision with RSD $\leq 3.8 \%$ for the three levels, with the exception of $7.6 \%$ for tangeretin. Also, good accuracy was attained for all flavonoids and phenolic acids (79.5-129.2\%), with some exceptions. Limit of detection ranged from 1 to $17 \mathrm{ng} \cdot \mathrm{g}^{-1}$ and limit of quantification ranged from 0.71 to $7.41 \mu \mathrm{g}$. Ruggedness was evident for most of the compounds considering all variations, and not observed in any situation for $p$-coumaric and ferulic acids, quercitrin and narirutin. Phenolic acids appeared to be most affected when formic acid was absent. Tangeretin was not affected when formic acid was absent and initial proportion of organic solvent was increased. Eriocitrin was only affected by decrease of initial organic solvent proportion and temperature.

\subsection{Phenolic compounds profile in Pêra-Rio orange juice}

Fig. 1 shows a typical chromatogram of Pêra-Rio orange juice extract obtained using the selected extraction and separation conditions, showing that good resolution was attained.

The phenolic compounds were identified (HPLC-DAD and HPLCMS) and quantified (HPLC-DAD) in the organic and conventional PêraRio orange juices. Identification criteria were (1) same retention time and DAD spectra as pure standards; (2) characteristic DAD spectra of compound class when pure standards were unavailable; (3) same $m / z$ and retention time as pure standards.

Table 2 shows the phenolic compounds identified in organic and conventional Pêra-Rio orange juices, retention time, chemical class based on experimental DAD spectra and maximum absorption wavelengths, and experimental m/z. Peaks 7, 10, 14 and 23 did not appear in the TIC chromatogram due to lack of ionization in either positive or negative modes. Three flavanones (eriocitrin, narirutin, hesperidin) and three flavones (rutin, nobiletin, and tangeretin) were identified according to criterion (1) and had the identity confirmed by MS (criterion 3 ) in all extracts from organic and conventional juices from the beginning, middle and end of the 2016 harvest. Also, 19 compounds tentatively identified in all juice extracts according to criterion (2) were described by chemical class: 7 acids, 6 flavanones and 6 flavones. All identified acids (peaks 1, 2, 3, 4, 13, 17 and 19) showed spectra characteristic of cinnamic acid derivatives, specifically ferulic or caffeic acid derivatives, from 270 to $370 \mathrm{~nm}$, with a band of higher intensity from 300 to 370, and another of lower intensity from 270 to $300 \mathrm{~nm}$ and maximum absorption between 325 and $330 \mathrm{~nm}$ (Gil-Izquierdo 


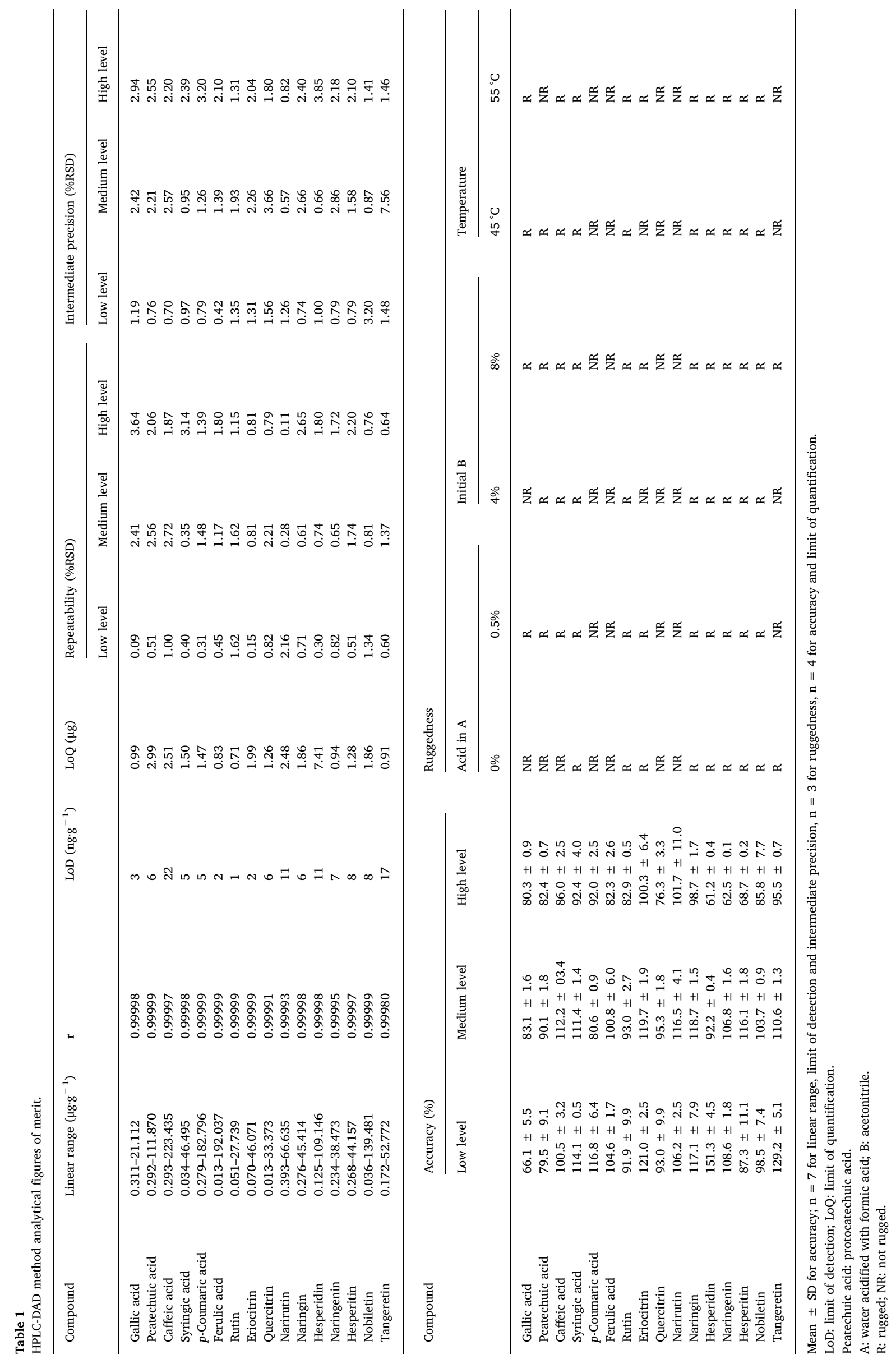




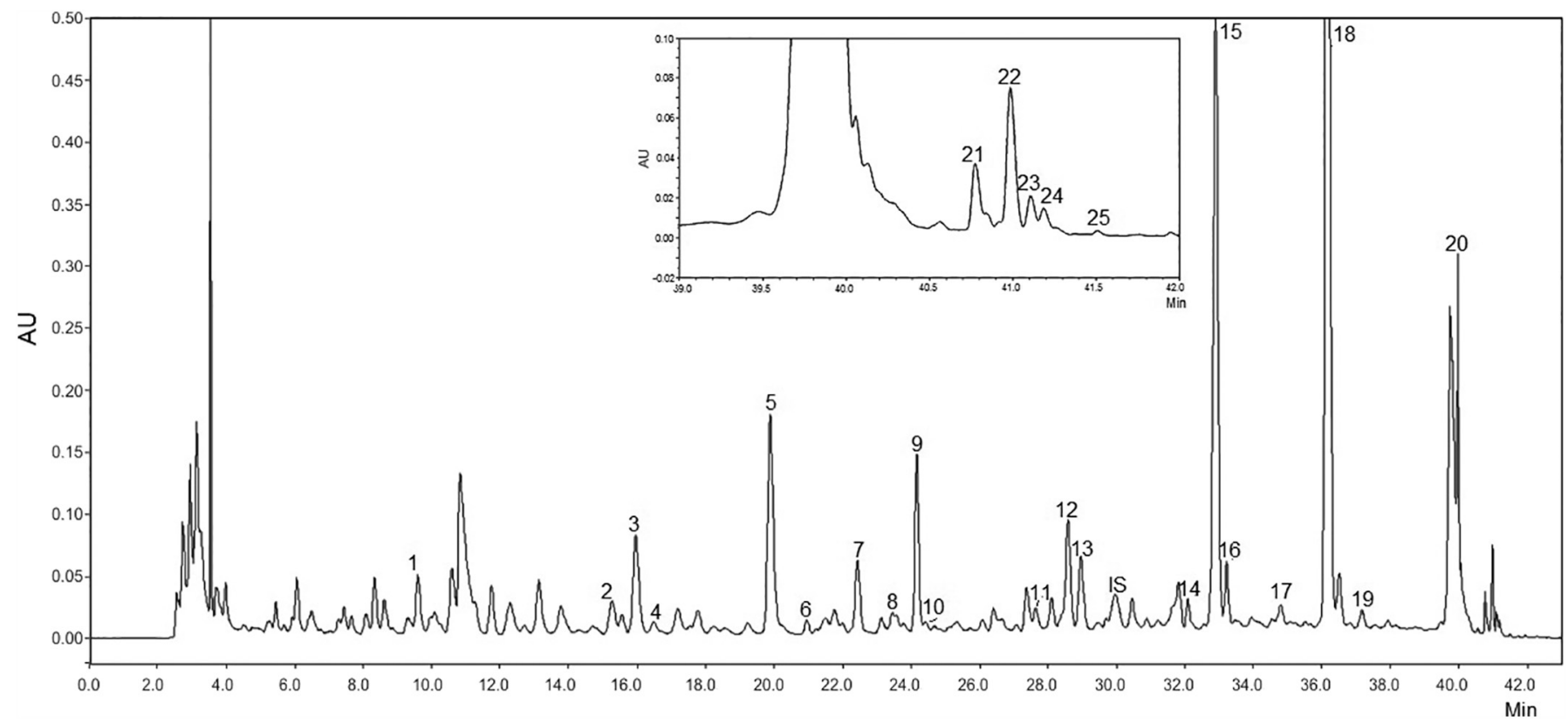

Fig. 1. Typical chromatogram of FCOJ extract at $280 \mathrm{~nm}$. Conditions as described in Sections 2.3 and 2.4. Peaks numbered according to Table 2.

et al., 2001). Flavanones (peaks 7, 8, 9, 14, 16 and 20) showed characteristic spectra with a band of high intensity from 250 to 300 , maximum absorption from 280 to $285 \mathrm{~nm}$, and a shoulder from 300 to 360 nm (Barreca, Gattuso, Laganà, Leuzzi, \& Belloco, 2016; Gattuso et al., 2007). Flavones from peaks 6, 10, 23 and 25 showed spectra with a band from 230 to 280 , maximum absorption at $255 \mathrm{~nm}$, and from 200 to $400 \mathrm{~nm}$, typical of flavones such as rutin. Peak 5 showed spectra similar to tangeretin, with a band from 240 to 280 and maximum absorption at $270 \mathrm{~nm}$, and from 300 to $360 \mathrm{~nm}$. Peak 21 showed spectra similar to nobiletin with three characteristic bands; two from 230 to 250 and 250 to $280 \mathrm{~nm}$, and a third of higher intensity from 290 to
$360 \mathrm{~nm}$ (He, Lian, Lin, \& Bernart, 1997) (Table 2). Identity confirmation of these compounds was not possible due to lack of standards available. Undetected compounds, if present, were below the limit of detection (Table 1).

Table 3 shows the levels of phenolic compounds from organic and conventional Pêra-Rio orange juices from the 2016 harvest. It is possible to observe that there were changes in the phenolic compounds levels in organic and conventional orange juices throughout the harvest; levels oscillated slightly amongst the juices. The major phenolic compounds in Pêra-Rio orange juice were hesperidin, narirutin and eriocitrin $\left(\geq 20 \mathrm{mg} \cdot 100 \mathrm{~g}^{-1}\right.$ ), and levels were more expressive in

Table 2

Phenolic compounds in organic and conventional orange juices from the 2016 harvest.

\begin{tabular}{|c|c|c|c|c|c|c|c|}
\hline \multirow[t]{2}{*}{ Peak } & \multirow[t]{2}{*}{ Compound } & \multirow[t]{2}{*}{$\mathrm{rt}(\min )$} & \multirow[t]{2}{*}{ Chemical class } & \multicolumn{3}{|c|}{ UV/Vis absorption (nm) } & \multirow[t]{2}{*}{$\mathrm{m} / \mathrm{z}$} \\
\hline & & & & $\lambda_{1}$ & $\lambda_{2}$ & $\lambda_{3}$ & \\
\hline 1 & ni & $9.6 \pm 0.0$ & Acid & 240 & 290 & 325 & 385 \\
\hline 2 & ni & $15.2 \pm 0.1$ & Acid & 240 & 290 & 324 & 399 \\
\hline 3 & ni & $15.9 \pm 0.0$ & Acid & 239 & 290 & 329 & 355 \\
\hline 4 & ni & $16.4 \pm 0.0$ & Acid & 240 & 290 & 325 & 399 \\
\hline 5 & ni & $19.9 \pm 0.0$ & Flavone & 270 & 335 & - & 163 \\
\hline 6 & ni & $21.0 \pm 0.0$ & Flavone & 254 & 353 & - & 787 \\
\hline 7 & ni & $22.4 \pm 0.0$ & Flavanone & 285 & 329 & - & - \\
\hline 8 & ni & $23.6 \pm 0.0$ & Flavanone & 284 & 350 & - & 398 \\
\hline 9 & ni & $24.2 \pm 0.0$ & Flavanone & 284 & 329 & - & 273 \\
\hline 10 & $\mathrm{ni}$ & $24.4 \pm 0.0$ & Flavone & 255 & 357 & - & - \\
\hline 11 & Rutin & $27.6 \pm 0.0$ & Flavone & 255 & 353 & - & 611 \\
\hline 12 & Eriocitrin & $28.6 \pm 0.0$ & Flavanone & 284 & 335 & - & 597 \\
\hline 13 & ni & $29.0 \pm 0.0$ & Acid & 240 & 290 & 323 & 561 \\
\hline 14 & ni & $32.1 \pm 0.0$ & Flavanone & 283 & 329 & - & - \\
\hline 15 & Narirutin & $32.9 \pm 0.0$ & Flavanone & 283 & 329 & - & 581 \\
\hline 16 & ni & $33.2 \pm 0.0$ & Flavanone & 283 & 327 & - & - \\
\hline 17 & ni & $34.8 \pm 0.1$ & Acid & 240 & 290 & 329 & 559 \\
\hline 18 & Hesperidin & $36.1 \pm 0.0$ & Flavanone & 284 & 328 & - & 611 \\
\hline 19 & ni & $37.2 \pm 0.0$ & Acid & 237 & 290 & 324 & 575 \\
\hline 20 & ni & $40.0 \pm 0.0$ & Flavanone & 281 & 335 & - & 714 \\
\hline 21 & ni & $40.8 \pm 0.0$ & Flavone & 240 & 270 & 326 & 373 \\
\hline 22 & Nobiletin & $41.0 \pm 0.0$ & Flavone & 247 & 271 & 329 & 403 \\
\hline 23 & ni & $41.1 \pm 0.0$ & Flavone & 252 & 270 & 334 & 433 \\
\hline 24 & Tangeretin & $41.2 \pm 0.0$ & Flavone & 271 & 317 & - & 373 \\
\hline 25 & ni & $41.5 \pm 0.0$ & Flavone & 255 & 318 & - & 403 \\
\hline
\end{tabular}

Mean \pm SD for retention time. 


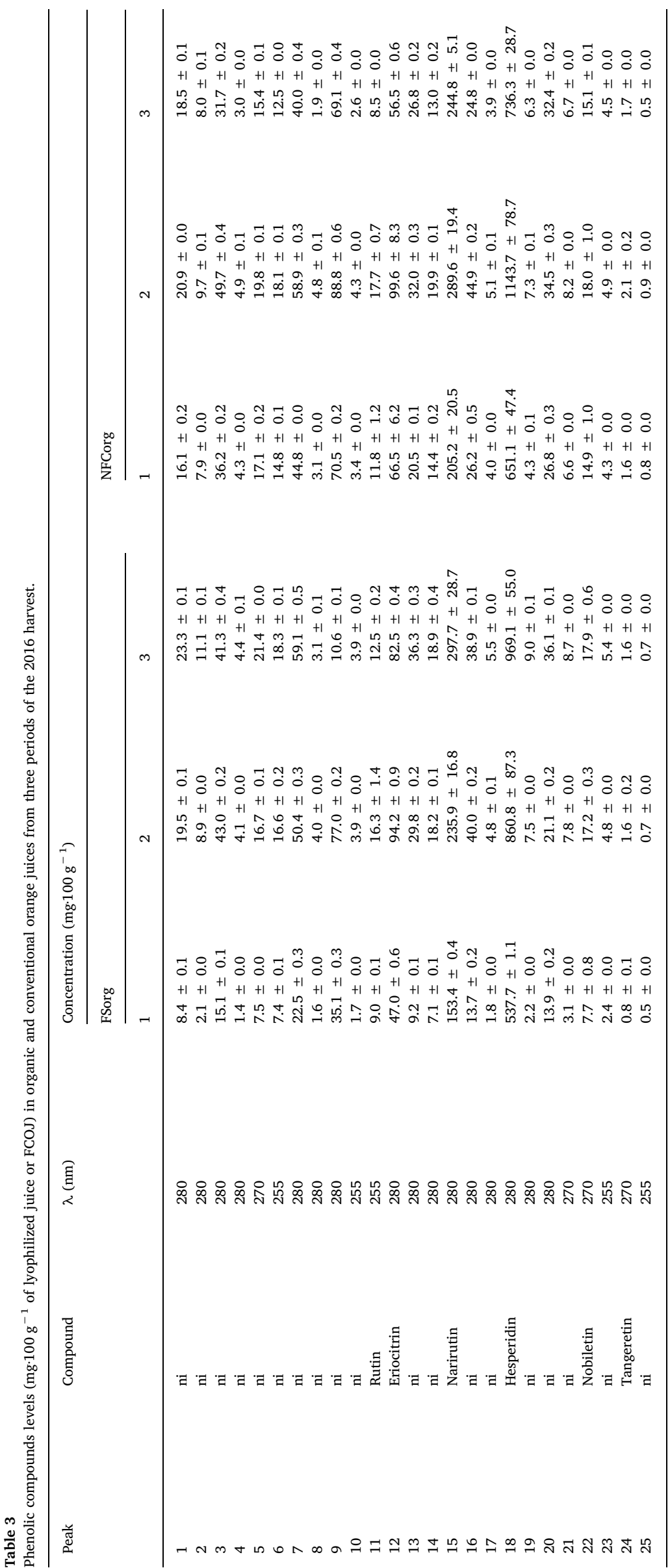

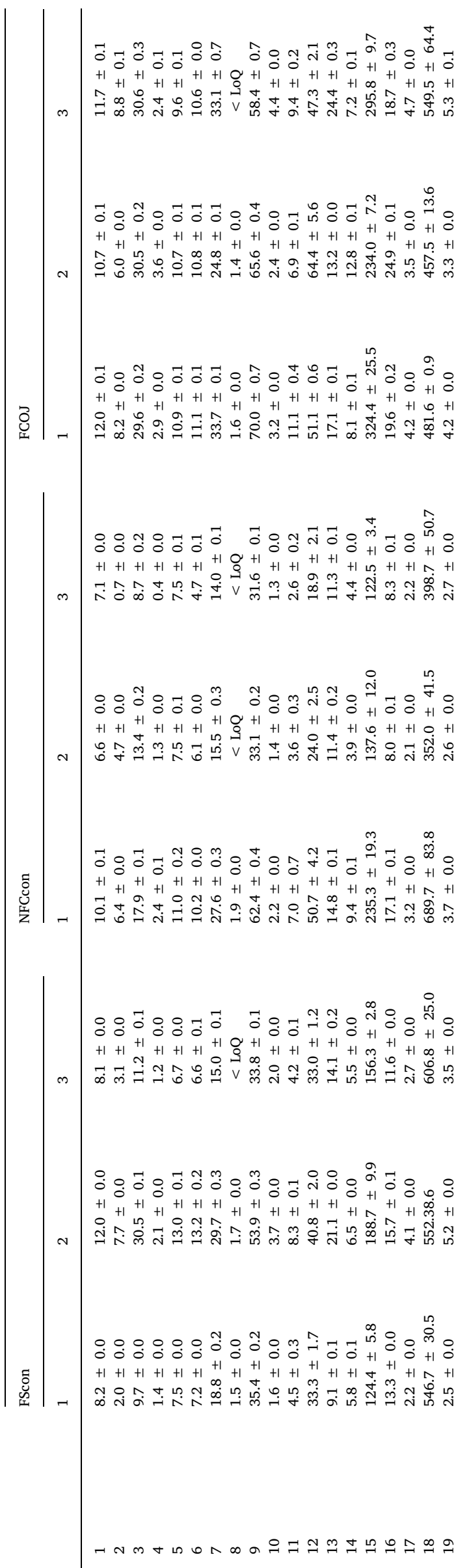




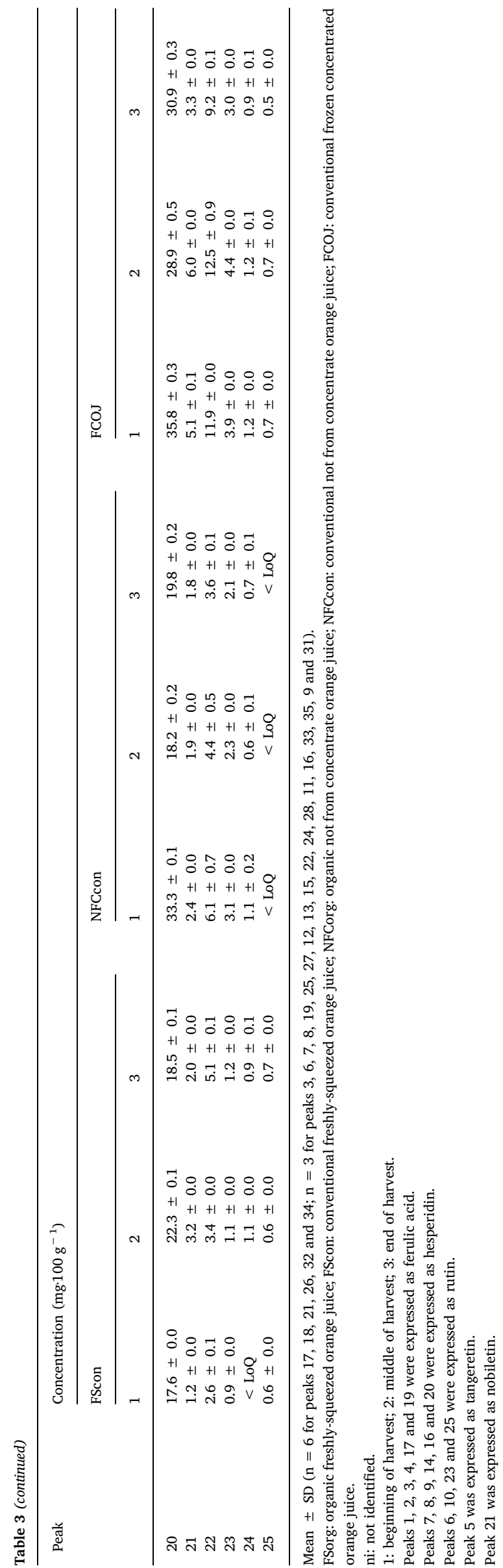

organic juices. Hesperidin levels were about 3 times higher than narirutin levels, and 10 times higher than eriocitrin levels in organic juices, 2-5 times higher than narirutin levels and 10-20 times higher than eriocitrin levels in conventional juices. Hesperidin levels increased during the harvest in organic FS and NFC juices, with a reduction at the end in NFC. Levels were about the same during the harvest in conventional FS and FCOJ, and reduced by half in NFC. Narirutin levels increased slightly at the beginning in organic FS and NFC juices and then were maintained. In conventional juices, narirutin levels increased at the beginning in FS and decreased in NFC, and then were maintained in both juices, while in FCOJ the levels were higher and practically the same during the harvest. Eriocitrin levels in organic juices increased at the beginning in both FS and NFC, were maintained in FS and decreased in NFC at the end of the harvest. In conventional juices, levels were maintained in FS and FCOJ, and decreased in NFC juice during the harvest (Table 3).

Minor compounds were represented by rutin, nobiletin and tangeretin. Rutin and nobiletin levels were 2-4 times higher in organic juices than in conventional juices, with the exception of FCOJ, which showed levels similar to organic juices. Rutin and nobiletin levels increased in organic FS at the beginning of the harvest; rutin levels were then decreased while nobiletin levels were maintained. In organic NFC, rutin levels increased at the beginning then decreased at the end, while nobiletin levels were maintained all through the harvest. Rutin levels in conventional FS juice showed the same movement as the organic FS, whereas nobiletin levels increased slightly. In conventional NFC and FCOJ, both rutin and nobiletin levels showed a reduction at the beginning, and then levels were maintained. Tangeretin levels in all juices were doubled at the beginning of the harvest and then were maintained in organic FS, while in NFC they were the same throughout the harvest. In conventional juices, levels increased at the beginning and were maintained in FS juice and FCOJ. Tangeretin levels were lower than the limit of quantification (Table 1) in conventional NFC and at the beginning of the harvest in conventional FS juice (Table 3).

Non-identified compounds were quantified as acids (peaks 1, 2, 3, 4, 13, 17 and 19) and expressed as ferulic acid, flavanones (peaks 7, 8, 9, 14, 16 and 20) expressed as hesperidin, flavones (peaks 6, 10, 23 and 25) expressed as rutin. Peak 5 was expressed as tangeretin, and peak 21 was expressed as nobiletin, according to the UV/Vis spectra similarity (Table 2). Organic juices showed higher levels of all phenolic compounds. In a general way, non-identified acid levels increased during the harvest for organic FS and NFC juices, while levels decreased in conventional FS and NFC juices and were about the same throughout the harvest in FCOJ. Flavanone levels oscillated in both organic FS and NFC juices; some compounds showed an increase in levels along the harvest, while others decreased. In conventional FS and FCOJ, levels were about the same during the harvest, with a slight decrease for some compounds. In conventional NFC juice, all flavanone levels decreased along the harvest. Peak 8 was below the limit of quantification (Table 1) in conventional FS from the end of the harvest, in conventional NFC from the middle and end of the harvest, and in FCOJ from the end of the harvest (Table 3).

The most important phenolic compounds in sweet orange and orange juices are flavanones hesperidin and narirutin (Gattuso et al., 2007; Khan et al., 2014; Peterson et al., 2006), especially in the Kozan, Moro, Tarocco and Sanguinello varieties (Kelebek et al., 2009; Leuzzi et al., 2000). Nobiletin, heptamethoxyflavone and tangeretin were also identified in Moro, Tarocco and Sanguinello orange juice (Leuzzi et al., 2000) and hand-squeezed and commercial orange juices from the Brazilian market (Pupin, Dennis, \& Toledo, 1998). Based on a thorough survey of the flavonoids of sweet orange juices from several varieties, Gattuso et al. (2007) highlighted hesperidin, narirutin, dydimin and eriocitrin as the main flavanones, while nobiletin, sinensetin, heptamethoxyflavone and tangeretin were the most important flavones. Our results from Pêra-Rio orange juices are in accordance to data presented in literature for hesperidin, narirutin, eriocitrin, nobiletin and 

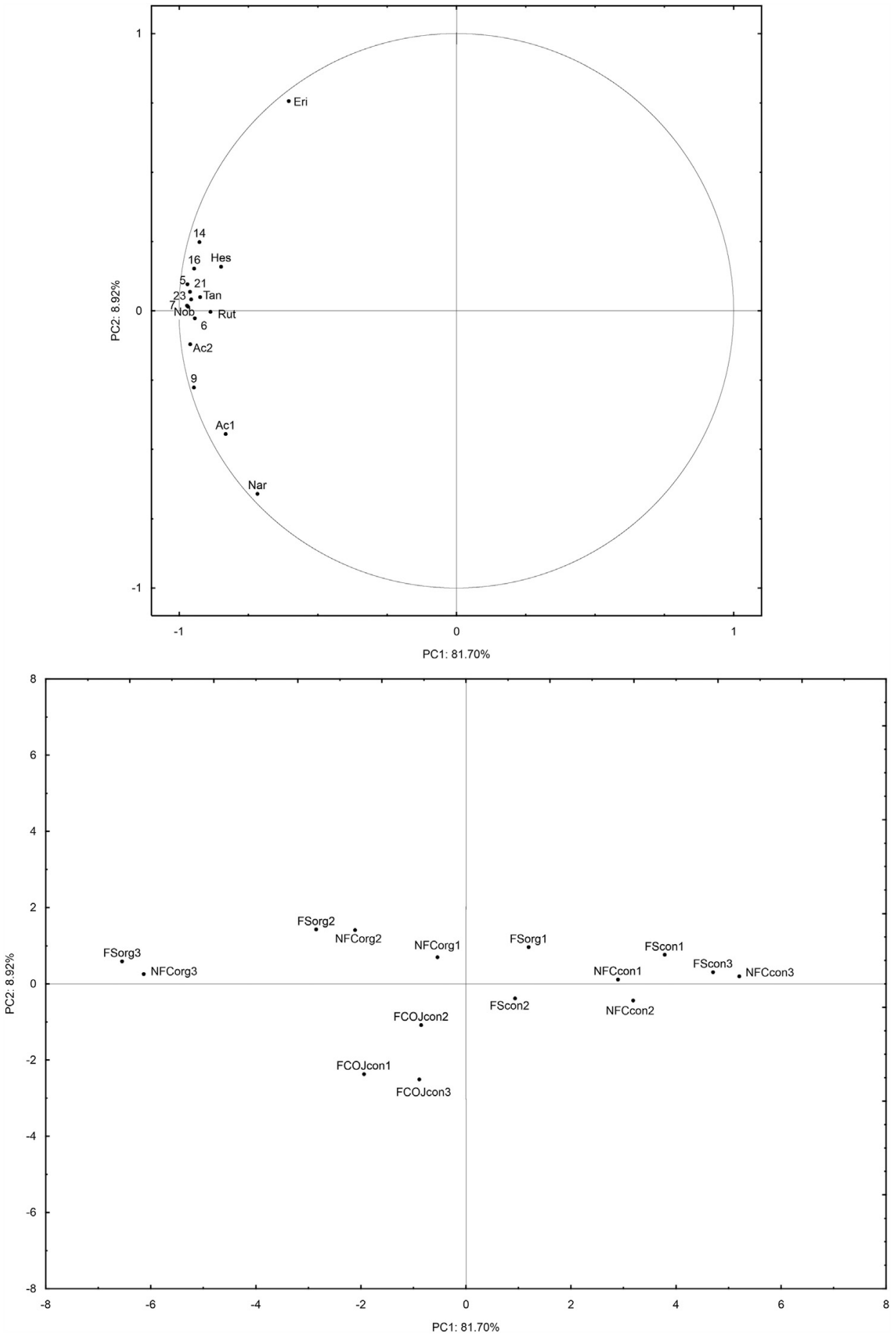

Fig. 2. Principal component analysis of phenolic compounds from organic (org) and conventional (con) Pêra-Rio orange juices from the beginning (1), middle (2) and end (3) of the 2016 harvest. FS: freshlysqueezed, NFC: not from concentrate, FCOJ: frozen concentrated orange juice. Compounds are numbered according to Table 2. Eri: eriocitrin, Hes: hesperidin, Rut: rutin, Nob: nobiletin, Tan: tangeretin, Nar: narirutin. tangeretin; dydimin, heptamethoxyflavone and sinensetin were not evaluated in our juices due to lack of standards available. It is worth mentioning that rutin, an important flavone for Pêra-Rio orange juice, was not reported by the abovementioned authors. The phenolic acids reported in literature (Rapisarda et al., 1999; Rapisarda, Carollo, Fallico, Tomaselli, \& Maccarone, 1998; Roussos, 2011) were not identified in our juices, though 7 compounds with spectra characteristic of caffeic or ferulic acid derivatives (Gil-Izquierdo et al., 2001) were found.

Principal component analysis (PCA) was used to represent data in two dimensions to differentiate orange juices and cultivation systems during the 2016 harvest. The phenolic compounds were represented by compound name or peak number, and chemical class when the sum of area values was used (Fig. 2). Acids were grouped in Acids 1 (peaks 2 and 17) and Acids 2 (peaks 1, 3, 4, 13 and 19). The first two principal components explained a variation of $90.62 \%$ of the levels of the phenolic compounds amongst organic and conventional juices during the harvest. PCA was suitable to differentiate the groups of compounds in all juices according to the spatial distribution. PC 1 allowed the differentiation of organic juices and conventional FCOJ from conventional FS and NFC juices, while PC 2 allowed the differentiation of conventional FCOJ from organic juices. PCA was able to differentiate organic juices from the middle and end of the harvest, as well as NFC from the beginning of the harvest, loaded negatively in PC 1 and positively in PC 2 , and conventional FCOJ, loaded negatively in PC 1 and in PC 2. PC 1 was mainly influenced (rotate component matrix $\geq 0.60$ ) by loading 
negatively the compounds: nobiletin $(-0.97)$, tangeretin $(-0.93)$, rutin $(-0.89)$, hesperidin $(-0.85)$, narirutin $(-0.72)$, eriocitrin $(-0.61)$ and non-identified compounds from peaks $7(-0.97), 5$ $(-0.97), 21(-0.96), 23(-0.96)$, acids 2 group $(-0.96), 9(-0.95)$, $16(-0.95), 6(-0.94), 14(-0.93)$ and acids 1 group $(-0.83)$. Organic juices from the end of the harvest were characterized by the higher levels of flavonoids hesperidin $(-0.85)$, tangeretin $(-0.93)$, nobiletin $(-0.97)$ and rutin $(-0.89)$, and non-identified flavanones and flavones $(\geq-0.93)$, whereas organic juices from the middle of the harvest were characterized by higher levels of eriocitrin $(-0.61)$. PC 2 was mainly influenced by loading negatively rutin, narirutin, acids 1 and 2 and peaks 6 and 9, and positively eriocitrin, hesperidin, nobiletin, tangeretin and peaks 5, 7, 14, 16, 21 and 23. The FCOJ was characterized by the higher levels of narirutin $(-0.72)$. Conventional FS and NFC were loaded positively in PC 1 and were characterized by lower levels of the phenolic compounds. Organic FS from the beginning of the harvest was loaded closer to conventional juices because its phenolic compounds levels were similar; phenolic compounds in organic Pêra-Rio oranges increased during the harvest (Table 3). PCA differentiated organic from conventional FS and NFC juices, and conventional FCOJ from conventional FS and NFC juices, thus differentiating cultivation and processing.

The results suggest that the higher levels of flavanones and flavones can be considered markers of organic juices of the 2016 harvest, when compared to conventional juices.

\section{Conclusion}

The developed HPLC-DAD method for the determination of the phenolic compounds profile of Pêra-Rio orange juice was validated, showing a wide linear range for the proposed application, good precision and accuracy, low limits of detection and quantification, and overall ruggedness. The extraction procedure using SPE also showed sufficient recovery for all phenolic compounds.

Hesperidin, narirutin, eriocitrin, nobiletin, rutin and tangeretin were identified and quantified in all organic and conventional juices, as well as non-identified cinnamic acid derivatives (7), flavanones (6) and flavones (6). There were changes in the phenolic compounds profile of organic and conventional orange juice during the harvest, and levels in organic juices were much higher than in conventional juices.

PCA clearly differentiated organic from conventional FS and NFC juices, and conventional FCOJ. Organic juices from the 2016 harvest were characterized by higher levels of flavonoids, and conventional FCOJ was characterized mainly by higher levels of narirutin.

\section{Acknowledgements}

Authors would like to thank Waters Technologies (São Paulo, SP, Brazil) for the support with LC-MS analysis, and Dr. Thaís Cesar from FCF/UNESP for providing some standards.

\section{Funding}

This work was funded by São Paulo Research Foundation (FAPESP) (Processes no. 2013/10138-0 and 2014/23303-1) and Brazilian Federal Agency for Support and Evaluation of Graduate Education-CAPES (scholarship grant of author E. Mesquita).

\section{Appendix A. Supplementary data}

Supplementary data to this article can be found online at https:// doi.org/10.1016/j.foodres.2017.12.025.

\section{References}

Abu-Reidah, I. M., Ali-Shtayeh, M., Jamous, R. M., Arráez-Román, \& Segura-Carretero, A.
(2015). HPLC-DAD-ESI-MS/MS screening of bioactive components from Rhus coriaria L. (Sumac) fruits. Food Chemistry, 166, 179-191.

Baldwin, E. A., Scott, J. W., Shewmaker, C. K., \& Schuch, W. (2000). Flavor trivia and tomato aroma: Biochemistry and possible mechanisms for control of important aroma components. Horticultural Science, 35(6), 1013-1022.

Barreca, D., Bellocco, E., Caristi, C., Leuzzi, U., \& Gattuso, G. (2011). Distribution of Cand $O$-glycosyl flavonoids, (3-hydroxy-3-methylglutaryl)glycosyl flavanones and furocoumarins in Citrus aurantium L. juice. Food Chemistry, 124, 576-582.

Barreca, D., Gattuso, G., Bellocco, E., Calderaro, A., Trombetta, D., Smeriglio, A., ... Nabavi, S. M. (2017). Flavanones: Citrus phytochemical with health-promoting properties. BioFactors, 43(4), 495-506.

Barreca, D., Gattuso, G., Laganà, G., Leuzzi, U., \& Belloco, E. (2016). C- and O-glycosyl flavonoids in Sanguinello and Tarocco blood orange (Citrus sinensis (L.) Osbeck) juice: Identification and influence on antioxidant properties and acetylcholinesterase activity. Food Chemistry, 196, 619-627.

Bilbao, M. L. M., Andrés-Lacueva, C., Jáuregui, O., \& Lamuela-Raventos, R. M. (2007). Determination of flavonoids in a Citrus fruit extract by LC-DAD and LC-MS. Food Chemistry, 101, 1742-1747.

Burin, V. M., Ferreira-Lima, N. E., Panceri, C. P., \& Bordignon-Luiz, M. T. (2014). Bioactive compounds and antioxidant activity of Vitis vinifera and Vitis labrusca grapes: Evaluation of different extraction methods. Microchemical Journal, 114, $155-163$.

Escriche, I., Kadar, M., Juan-Borrás, M., \& Domenech, E. (2011). Using flavonoids, phenolic compounds and headspace volatile profile for botanical authentication of lemon and orange honeys. Food Research International, 44, 1504-1513.

Fang, X., Wang, J., Hao, J., \& Guo, N. (2009). Simultaneous extraction, identification and quantification of phenolic compounds in Eclipta prostrata using microwave-assisted extraction combined with HPLC-DAD-ESI-MS/MS. Food Chemistry, 188, 527-536.

FAO. Food and Agriculture Organization (2003). World markets for organic citrus and citrus juices. FAO community and trade policy research working paper.

Fundecitrus (2017). Fundo de Defesa da Citricultura. Revista do citricultor. Retrieved from http://www.fundecitrus.com.br/comunicacao/revista/revista-42/50, Accessed date: 7 October 2017.

Gattuso, G., Barreca, D., Gargiulli, C., Leuzzi, U., \& Caristi, C. (2007). Flavonoid composition of citrus juices. Molecules, 12, 1641-1673.

Gil-Izquierdo, A., Gil, M. I., Ferreres, F., \& Tomás-Barberán, F. A. (2001). In vitro availability of flavonoids and other phenolics in orange. Journal of Agriculture and Food Chemistry, 49, 1035-1041.

Haard, N. F. (1984). Postharvest physiology and biochemistry of fruits and vegetables. Journal of Chemical Education, 61(4), 277-283.

He, X., Lian, L., Lin, L., \& Bernart, M. W. (1997). High-performance liquid chromatography-electrospray mass spectrometry in phytochemical analysis of sour orange (Citrus aurantium L.). Journal of Chromatography A, 791, 127-134.

ICH (2005). Validation of analytical procedures: Text and methodology. ICH Tripartite guideline. International conference on harmonisation of technical requirements for registration of pharmaceuticals for human use. Chicago, USA.

IFOAM. International Federation of Organic Agriculture Movements (2015). Consolidated annual report of IFOAM - Organics International.

Janzantti, N. S., Santos, G. C., \& Monteiro, M. (2012). Shelf life of fresh and pasteurized organic passion fruit (Passiflora edulis F. flavicarpa deg) pulp. Journal of Food Processing and Preservation, 38, 262-270.

Kelebek, H., Selli, S., Canbas, A., \& Cabaroglu, T. (2009). HPLC determination of organic acids, sugars, phenolic compositions and antioxidant capacity of orange juice and orange wine made from a Turkish cv. Kozan. Microchemical Journal, 91, 187-192.

Khan, M. K., Huma, Z. E., \& Dangles, O. (2014). A comprehensive review on flavanones, the major citrus polyphenols. Journal of Food Composition and Analysis, 33, 85-104.

Leuzzi, U., Caristi, C., Panzera, V., \& Licandro, G. (2000). Flavonoids in pigmented orange juice and second-pressure extracts. Journal of Agriculture and Food Chemistry, 48, 5501-5506.

López-Cobo, A., Gómez-Caravaca, A. M., Svarc-Gajíc, J., Segura-Carretero, A., \& Fernández-Gutiérrez, A. (2015). Determination of phenolic compounds and antioxidant activity of a Mediterranean plant: The case of Satureja montana subsp. kitaibelii. Journal of Functional Foods, 18, 1167-1178.

Macoris, M. S., De Marchi, R., Janzantti, N. S., \& Monteiro, M. (2011). The influence of ripening stage and cultivation system on the total antioxidant activity and total phenolic compounds of yellow passion fruit pulp. Journal of the Science of Food and Agriculture, 92, 1886-1891.

Macoris, M. S., Janzantti, N. S., Garruti, D. S., \& Monteiro, M. (2011). Volatile compounds from organic and conventional passion fruit (Passiflora edulis F. Flavicarpa) pulp. Ciência e Tecnologia de Alimentos, 31(2), 430-435.

Magnusson, B., \& Örnemark, U. (2014). The fitness for purpose of analytical methods - A laboratory guide to method validation and related topics (2 ed.). Eurachem.

Martini, S., Conte, A., \& Tagliazucchi, D. (2017). Phenolic compounds profile and antioxidant properties of six sweet cherry (Prunus avium) cultivars. Food Research International, 97, 15-26.

Medina-Remon, A., Estruch, R., Tresserra-Rimbau, A., Vallverdú-Queralt, A., \& LamuelaRaventos, R. M. (2013). The effect of polyphenol consumption on blood pressure. Mini-Reviews in Medicinal Chemistry, 13, 1137-1149.

Neves, M. F., Trombin, V. G., Milan, P., Lopes, F. F., Cressoni, F., \& Kalaki, R. (2010). O Retrato da Citricultura Brasileira. São Paulo: Markestrat.

Peterson, J. J., Dwyer, J. T., Beecher, G. R., Bhagwat, S. A., Gebhardt, S. E., Haytowitz, D. B., \& Holden, J. M. (2006). Flavanones in oranges, tangerines (mandarins), tangors, and tangelos: A compilation and review of the data from the analytical literature. Journal of Food Composition and Analysis, 19, 66-73.

Pinto, M. S., Lajolo, F. M., \& Genovese, M. I. (2008). Bioactive compounds and quantification of total ellagic acid in strawberries (Fragaria $\times$ ananassa Duch.). Food 
Chemistry, 107, 1629-1635.

Pupin, A. M., Dennis, M. J., \& Toledo, M. C. F. (1998). Polymethoxylated flavones in Brazilian orange juice. Food Chemistry, 63(4), 513-518.

Rapisarda, P., Carollo, G., Fallico, B., Tomaselli, F., \& Maccarone, E. (1998). Hydroxycinnamic acids as markers of italian blood orange juices. Journal of Agriculture and Food Chemistry, 46, 464-470.

Rapisarda, P., Tomaino, A., Lo Cascio, R., Bonina, F., De Pasquale, A., \& Saija, A. (1999). Antioxidant effectiveness as influenced by phenolic content of fresh orange juices. Journal of Agriculture and Food Chemistry, 47, 4718-4723.

Rojas-Garbanzo, C., Zimmermann, B. F., Schulze-Kaysers, N., \& Schieber, A. (2016). Characterization of phenolic and other polar compounds in peel and flesh of pink guava (Psidium guajava L. cv. 'Criolla') by ultra-high performance liquid chromatography with diode array and mass spectrometry detection. Food Research International. http://dx.doi.org/10.1016/j.foodres.2016.12.004.

Roussos, P. A. (2011). Phytochemicals and antioxidant capacity of orange (Citrus sinensis (1.) Osbeck cv. Salustiana) juice produced under organic and integrated farming system in Greece. Scientia Horticulturae, 120, 253-258.

Santos, G., \& Monteiro, M. (2004). Sistema orgânico de produção de alimentos. Revista de
Alimentos e Nutrição, 15, 73-86.

Thompson, M., Ellison, S. L. R., \& Wood, R. (2002). Harmonized guidelines for singlelaboratory validation of methods of analysis (IUPAC technical report). Journal of Pure and Applied Chemistry, 74(5), 835-855.

Tripoli, E., La Guardia, M., Giammanco, S., Di Majo, D., \& Giammanco, M. (2007). Citrus flavonoids: Molecular structure, biological activity and nutritional properties: A review. Food Chemistry, 104, 466-479.

USDA. United States Department of Agriculture (2016). Brazil: Citrus annual. https:// www.fas.usda.gov/data/brazil-citrus-annual-1, Accessed date: 26 September 2017.

Vallverdú-Queralt, A., Arranz, S., Medina-Remón, A., Casals-Ribes, I., \& LamuelaRaventos, R. M. (2011). Changes in phenolic content of tomato products during storage. Journal of Agricultural and Food Chemistry, 59, 9358-9365.

Vallverdú-Queralt, A., Jáuregui, O., Medina-Remon, A., \& Lamuela-Raventos, R. M. (2012). Evaluation of a method to characterize the phenolic profile of organic and conventional tomatoes. Journal of Agricultural and Food Chemistry, 60, 3373-3380.

Zou, Z., Xi, W., Hu, Y., Nie, C., \& Zhou, Z. (2016). Antioxidant activity of Citrus fruits. Food Chemistry, 196, 885-896. 